\title{
Analyst
}

Check for updates

Cite this: Analyst, 2019, 144, 1292

\section{Dual-template molecularly imprinted polymers for dispersive solid-phase extraction of fluoroquinolones in water samples coupled with high performance liquid chromatography $\dagger$}

\author{
Wenhui Lu, ${ }^{a, c}$ Jie Liu, ${ }^{\text {b,c }}$ Jinhua Li, ${ }^{c}$ Xiaoyan Wang, ${ }^{c}$ Min Lv, ${ }^{c}$ Rong Cui*b and \\ Lingxin Chen iD *c,d
}

\begin{abstract}
Novel dual-template molecularly imprinted polymers (dt-MIPs) were prepared by simple and facile precipitation polymerization using norfloxacin (NOR) and enrofloxacin (ENR) as templates for simultaneous selective recognition and extraction of the two fluoroquinolones (FQs). The as-prepared dt-MIPs exhibited high adsorption capacity and excellent selectivity towards NOR and ENR. Several main parameters affecting the efficiency of dt-MIP based dispersive solid-phase extraction (DSPE) were systematically investigated, coupled with high performance liquid chromatography determination. Consequently, high enrichment factors of 71 and 61 were obtained for NOR and ENR respectively, and good linearity in the range of $1-200 \mathrm{~g} \mathrm{~L}^{-1}$ was observed, with correlation coefficients $(r)$ above 0.9977 . The limits of detection and quantification for NOR were 0.22 and $0.67 \mu \mathrm{g} \mathrm{L}^{-1}$, respectively, and 0.36 and $0.98 \mu \mathrm{g} \mathrm{L}{ }^{-1}$ for ENR. Satisfactory recoveries of the two FQs from spiked lake, sea and tap water samples at three concentration levels were attained in the range of $80.9-101.0 \%$ with relative standard deviations of $0.9-6.9 \%$. The present study not only has great potential for applications in $F Q$ determination, but will also enrich research into dual/multi-template imprinting.
\end{abstract}

Received 5th November 2018 Accepted 4th December 2018 DOI: $10.1039 / c 8 a n 02133 c$ rsc.li/analyst terial resistance, poison plants and aquatic organisms, and have an adverse effect on human health. ${ }^{3}$ Recent research has also revealed that a multitude of $\mathrm{FQs}$ have been detected at levels of ng $\mathrm{L}^{-1}$ to $\mu \mathrm{g} \mathrm{L}^{-1}$ in environmental water systems, and in aquatic and terrestrial organisms. ${ }^{4,5}$ Therefore, sensitive, efficient and selective analytical methods are required to determine trace levels of FQs in complicated matrices.

Currently available instrumental analytical technologies for FQ determination include high performance liquid chromatography (HPLC) coupled with a fluorescence detector (FLD), ${ }^{6}$ mass spectrometry (MS), ${ }^{7}$ an ultraviolet (UV) detector ${ }^{8}$ or diode array detector (DAD), ${ }^{9}$ and capillary electrophoresis (CE) ${ }^{10}$ Given the low concentration levels of FQs in samples, a number of pretreatment technologies have been proposed to extract and enrich FQs before instrumental analysis, such as solid-phase extraction (SPE) ${ }^{11}$ dispersive solid-phase extraction (DSPE), ${ }^{12}$ solid-phase microextraction, ${ }^{13}$ matrix solidphase dispersion, ${ }^{14}$ stir-bar sorptive extraction, ${ }^{15}$ stir-cake sorptive extraction, ${ }^{16}$ hollow fiber based liquid-phase microextraction, ${ }^{17}$ and dispersive liquid-liquid microextraction. ${ }^{18}$ Amongst them, SPE is commonly used in food, ${ }^{19}$ environmental $^{20}$ and biological ${ }^{21}$ fields due to its high extraction efficiency and reusability. Recent studies have focused on the innovation of the SPE method and the preparation and appli- 
cation of novel adsorbents. ${ }^{19-24}$ DSPE is based on SPE technology, and sorbents are directly added into the sample without packing (into a column) and conditioning, which is simpler, more efficient and time-saving. ${ }^{22}$ He et al. used commercial magnetic graphene as a DSPE sorbent combined with HPLC for the extraction and determination of FQ residues in foods of animal origin. ${ }^{23}$ Amoli-Diva et al. synthesized magnetic multi-walled carbon nanotube nanocomposites as adsorbents for dispersive micro-solid-phase extraction coupled with surfactant-enhanced spectrofluorimetric detection for the enrichment and determination of ofloxacin and lomefloxacin in biological and environmental samples. ${ }^{24}$

Besides, molecularly imprinted polymers (MIPs) with predictable structures, specific recognition abilities and universal applications have become attractive adsorbents and have been widely applied in sample pretreatment and chromatographic separation to specifically extract the template molecules from complex matrices. ${ }^{25-27}$ MIPs have also been used for SPE of FQs in various samples. For example, Turiel et al. synthesized MIPs in one single preparation step by precipitation polymerization using ciprofloxacin (CIP) as a template, and successfully employed them both as SPE sorbents and as a stationary phase in the determination of FQs in soil samples. ${ }^{28} \mathrm{Wu}$ and a coworker $^{29}$ used levofloxacin (LEV) as a template and ionic liquids as reaction solvents to synthesize MIPs by bulk polymerization based on molecular crowding, and applied them as SPE sorbents followed by HPLC to enrich and determine FQs in milk and lake water samples. Urraca et al. ${ }^{30}$ prepared MIPs using enoxacin (ENOX) as a template by surface imprinting on silica beads, which were packed into an SPE cartridge coupled with HPLC-FLD and HPLC-MS/MS for the selective extraction and detection of six FQs in chicken muscle samples. In the above mentioned MIPs, the specific recognition sites for the template are created by a single template. However, the imprinting process does not have to be limited to a single template for practical applications. ${ }^{31}$ Although single-template MIPs have also been used to extract structural analogues of the template molecule through cross-selectivity, sometimes the prepared MIPs cannot effectively bind the structural analogues, thus diminishing their affinity toward analytes. $^{32}$ Accordingly, a multi-template imprinting strategy has been proposed and increasingly used to synthesize MIPs with multiple types of recognition sites in one format, using dual/multiple targets/species as templates, which can widen the applications of MIPs for simultaneous recognition and extraction of more than one analyte. ${ }^{33-40}$ For instance, Liu et $a{ }^{36}{ }^{36}$ adopted a multi-template imprinting strategy using melamine and dicyandiamide as templates to prepare MIPs, which were packed into an SPE column to simultaneously extract melamine and dicyandiamide from powdered milk. Compared with single-template MIPs and non-imprinted polymers, the dual-template MIPs (dt-MIPs) showed better affinity and selectivity for the two templates. Jafari et $a l .{ }^{38}$ synthesized magnetic dt-MIPs using acetaminophen and codeine as templates by combining sol-gel polymerization and surface imprinting techniques, and then used them as magnetic SPE
(MSPE) adsorbents for the simultaneous enrichment of acetaminophen and codeine in urine samples. Xia et $a{ }^{40}{ }^{40}$ synthesized dt-MIPs using a mixture of clonazepam and 2-(trifluoromethyl)phenothiazine as templates via precipitation polymerization, and then used the dt-MIPs to prepare an SPE cartridge for the simultaneous extraction of two classes of sedatives ( 4 phenothiazines and 5 benzodiazepines) in swine complete formula feed samples.

Inspired by these studies, herein we chose two typical FQs as templates, namely norfloxacin (NOR) and enrofloxacin (ENR), which are often used in human and veterinary medicine, to synthesize novel dt-MIPs by simple facile precipitation polymerization for the concurrent recognition and extraction of these two FQs. Indexing of NOR and ENR can be used to evaluate the possible migration routes or spatial distribution of residual FQs in the environment, and as far as we are aware no reported studies have used them as templates for dt-MIPs. The as-prepared dt-MIPs were well characterized and assessed, and utilized as DSPE adsorbents followed by HPLC determination. To the best of our knowledge, this is the first demonstration of the extraction of FQs by dt-MIP based DSPE. The conditions for the dt-MIP based DSPE, including dt-MIP dosage, sample $\mathrm{pH}$, extraction time, type and volume of desorption solvent and desorption time, were optimized. Subsequently, the dt-MIPs-DSPE-HPLC method was validated and successfully applied in real water sample analysis.

\section{Experimental}

\section{Reagents and materials}

Norfloxacin (NOR, 99\%), enrofloxacin (ENR, 98.5\%), ciprofloxacin (CIP, 99\%), pefloxacin (PEF, 99\%) and danofloxacin (DAN, 98\%) were purchased from Dalian Meilun Biological Technology Co., Ltd (Dalian, China), and enoxacin (ENO, 98\%) was obtained from Shanghai Macklin Biochemical Co., Ltd (Shanghai, China). Standard solutions of ENR in methanol with concentration of $100 \mathrm{mg} \mathrm{L}^{-1}$ and NOR in ethanol with concentration of $100 \mathrm{mg} \mathrm{L}^{-1}$ were purchased from AgroEnvironmental Protection Institute, Ministry of Agriculture (Tianjin, China) and stored at $4{ }^{\circ} \mathrm{C}$ in a refrigerator before use. HPLC grade acetonitrile and formic acid were obtained from Sigma-Aldrich (Shanghai, China). Methacrylic acid (MAA), 4-vinylpyridine (4-VP) and ethylene glycol dimethacrylate (EGDMA) were purchased from Sigma-Aldrich and purified prior to use in order to remove stabilizers. Acrylamide (AAm) was purchased from Aladdin (Shanghai, China) and recrystallized in water prior to use. 2,2'-Azo-bis-isobutyronitrile (AIBN) was obtained from Aladdin (Shanghai, China), and was recrystallized in ethanol prior to use. Methanol, acetonitrile and acetic acid were obtained from Sinopharm Chemical Reagent Co., Ltd (Shanghai, China).

Ultrapure water with a specific resistance of $18.2 \mathrm{M} \Omega \mathrm{cm}$ was produced by a Pall Cascada ${ }^{\mathrm{TM}}$ lab water purification system (Pall Corp., USA) for aqueous solution preparation 
throughout the study. All other reagents were analytical reagent grade and used without further purification steps.

\section{Instrumentation}

Analyses were performed using an Agilent 1260 Infinity HPLC system (Agilent Technologies, Palo Alto, CA, USA) equipped with a diode array detector (DAD). Optimized fluoroquinolone separation was achieved in a reversed-phase column (Agilent ZORBAX SB-C18, $150 \times 4.6 \mathrm{~mm}$ i.d., particle size: $5 \mu \mathrm{m}$ ) by isocratic elution using a mobile phase consisting of water (containing $0.05 \%$ formic acid)-acetonitrile $(85: 15, \mathrm{v} / \mathrm{v})$ at a flow

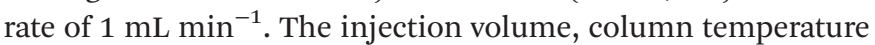
and detection wavelength were individually set as $20 \mu \mathrm{L}, 30^{\circ} \mathrm{C}$ and $280 \mathrm{~nm}$.

The morphologies of the polymer particles were examined by scanning electron microscopy (SEM, Hitachi S-4800, Japan). All samples were sputter-coated with thin gold film before observation. The size distributions of the MIPs and nonimprinted polymers (NIPs) were tested using a laser particle analyzer (Malvern Mastersizer 2000F, UK). Infrared spectra of samples were obtained using a Fourier transform infrared (FT-IR) spectrometer (Thermo Nicolet Corporation, USA) by using a pressed $\mathrm{KBr}$ tablet. The thermal stability of the MIPS was measured by thermogravimetric analysis (TGA) using a Mettler 5MP/PF7548/MET/400 W thermal analyzer (Mettler Toledo, Switzerland) in a nitrogen atmosphere between 40 and $750{ }^{\circ} \mathrm{C}$ with an nitrogen flow rate of $50 \mathrm{~mL} \mathrm{~min}^{-1}$ and a heating rate of $20^{\circ} \mathrm{C} \mathrm{min}^{-1}$, in order to obtain TGA and derivative thermogravimetry (DTG) data. Specific surface areas were obtained using the Brunauer-Emmett-Teller (BET) method and pore diameters were determined using the Barrett-JoynerHalenda (BJH) method, which were performed on a Full-automatic Specific Surface Instrument (3H-2000PS4, Beishide Instrument Technology, China).

\section{Preparation of dt-MIPs}

dt-MIPs were prepared according to a non-covalent approach by a simple facile precipitation polymerization using NOR and ENR as the templates, and the schematic procedure is shown in Fig. 1A. Briefly, NOR (0.1 mmol), ENR (0.1 mmol), and MAA
$(0.8 \mathrm{mmol})$ were dissolved in acetonitrile $(29 \mathrm{~mL})$ and methanol $(1 \mathrm{~mL})$ in a $100 \mathrm{~mL}$ glass flask, and were pre-polymerized at $4{ }^{\circ} \mathrm{C}$ in the dark for $12 \mathrm{~h}$. The cross-linker (EGDMA, $4 \mathrm{mmol}$ ) and the initiator (AIBN, $20 \mathrm{mg}$ ) were then successively added to the solution. The solution was sonicated for $5 \mathrm{~min}$ and deoxygenated with nitrogen for $15 \mathrm{~min}$ to remove dissolved oxygen. Then, the flask was sealed under a nitrogen atmosphere and placed in a water bath. The temperature of the water bath was increased from room temperature to $60^{\circ} \mathrm{C}$ over approximately $2 \mathrm{~h}$ and then kept at $60^{\circ} \mathrm{C}$ for $24 \mathrm{~h}$ for the polymerization. After the polymerization, the polymers were washed with acetonitrile to remove the unreacted reagents and then washed with methanol/acetic acid (90:10, v/v) to remove the template molecules until no template was detected by HPLC. Finally, the polymer particles were washed with methanol to remove acetic acid and dried under vacuum at $40{ }^{\circ} \mathrm{C}$ before use. As a control, the corresponding NIPs were synthesized using the same conditions in the absence of the template molecules.

\section{Adsorption experiments}

Static adsorption experiments were carried out to investigate the adsorption capacity of the synthesized polymers. In short, $5.0 \mathrm{mg}$ dt-MIPs or NIPs were dispersed in $10 \mathrm{~mL}$ plastic centrifuge tubes containing $4 \mathrm{~mL}$ ENR and NOR solutions of various

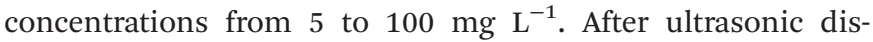
persion, the mixtures were shaken for $24 \mathrm{~h}$ at room temperature. After centrifugation and filtration through $0.22 \mu \mathrm{m}$ cellulose acetate membrane filters, the supernatant was collected and tested by HPLC. The adsorption capacity $(Q)$ was calculated according to the following equation:

$$
Q=\frac{\left(C_{0}-C_{\mathrm{e}}\right) V}{m}
$$

where $C_{0}\left(\mathrm{mg} \mathrm{L}^{-1}\right)$ and $C_{\mathrm{e}}\left(\mathrm{mg} \mathrm{L}^{-1}\right)$ are the initial and equilibrium concentrations of the template compounds, respectively. $V(\mathrm{~mL})$ is the volume of the solution, and $m(\mathrm{~g})$ is the mass of dt-MIPs or NIPs.

According to the static adsorption procedure, the specific adsorption performances of the dt-MIPs were investigated
A
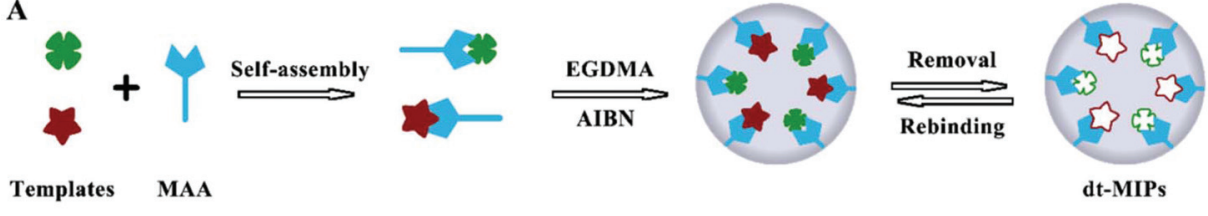

B
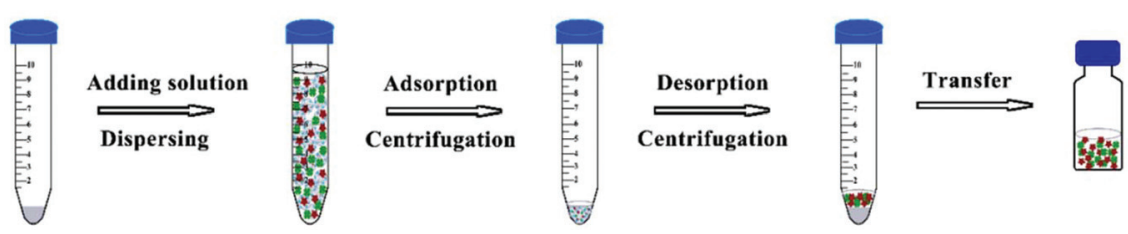

Fig. 1 Schematic procedures for the preparation of dt-MIPs by precipitation polymerization (A) and dt-MIPs-DSPE (B). 
using a mixed solution of NOR, ENR and other four analogues (PEF, DAN, ENO and CIP) at individual concentration levels of $80 \mathrm{mg} \mathrm{L}{ }^{-1}$. The imprinting factor $(\alpha)$, distribution coefficient $\left(K_{\mathrm{d}}\right)$, selectivity coefficient $(K)$ and relative selectivity coefficient $\left(K^{\prime}\right)$ were used to evaluate the selectivity of the dt-MIPs. The related descriptions and equations are given in Experimental S1. $\dagger$

\section{DSPE procedure and real sample preparation}

The dt-MIPs-DSPE procedure is schematically shown in Fig. 1B. Briefly, $10 \mathrm{mg}$ dt-MIPs were added into a centrifugation tube containing $10 \mathrm{~mL}$ NOR and ENR solutions at concentrations of $50 \mu \mathrm{g} \mathrm{L} \mathrm{L}^{-1}$. The dt-MIPs were dispersed by ultrasonication for $2 \mathrm{~min}$, and then the tube was continuously shaken for $3 \mathrm{~h}$ at room temperature. After centrifugation at $9000 \mathrm{rpm}$ for $10 \mathrm{~min}$ and discarding the supernatant, the polymers and adsorbed target molecules at the bottom of the tube were dried in a vacuum drying oven. Then, $150 \mu \mathrm{L}$ methanol/ acetic acid (90:10, v/v) were added and the analytes were desorbed by ultrasound for $5 \mathrm{~min}$, followed by centrifugation at $9000 \mathrm{rpm}$ for $10 \mathrm{~min}$ and collection of the supernatant. Finally, $20 \mu \mathrm{L}$ of the desorption solvent was injected into the HPLC system for analysis after filtration through $0.22 \mu \mathrm{m}$ organic nylon membrane filters.

Tap water was collected in the laboratory after it had flowed for about $5 \mathrm{~min}$. Surface seawater was obtained from Haichang Fisherman's Wharf at the Yellow Sea located in the coastal zone area of Yantai City, and lake water was collected from an artificial lake named Sanyuan Lake located in the school yard of Yantai University. All water samples were stored at $4^{\circ} \mathrm{C}$ and filtered through a $0.22 \mu \mathrm{m}$ hydrophilic polypropylene membrane for the removal of possible suspended impurities prior to use.

\section{Results and discussion}

\section{Preparation of dt-MIPs using NOR and ENR}

The dt-MIPs were synthesized by precipitation polymerization using NOR and ENR as templates, which are typical FQs commonly used in human and veterinary applications. In order to synthesize dt-MIPs with homogeneous morphology and good adsorption and recognition properties, typical parameters should be optimized, such as type and concentration of porogen, functional monomer and cross-linker. Generally, porogens act as both solvents and pore forming agents in the polymerization process. Especially in non-covalent interaction systems, porogens can also influence the bonding strength between the monomer and the template, and the properties and morphology of the imprinted polymers. ${ }^{41}$ In this study, methanol, acetonitrile and chloroform were used as porogens for the preparation of dt-MIPs. It was found that soft bulk polymers were obtained when using methanol and chloroform as porogens. Because the solubility of NOR in methanol was better than that in acetonitrile, a suitable volume of methanol was added when using acetonitrile as the porogen, and consequently imprinted polymers with uniform morphology were obtained. Moreover, the effect of volume of porogen (aceto- nitrile containing methanol) was also investigated and the results indicated that the obtained dt-MIPs had comparatively higher adsorption capacity and more uniform morphology when the total volume of porogen was $30 \mathrm{~mL}$ (acetonitrile/ methanol $=29: 1, \mathrm{v} / \mathrm{v}$ ). The functional monomer plays an important role in providing functional groups which can form a pre-polymerization complex with the template by covalent or non-covalent interactions. ${ }^{41,42}$ MAA, AAm and 4-VP were chosen as monomers and the results showed that it was easier to obtain more uniform spherical polymers with higher adsorption capacity when using MAA as monomer than when using AAm or 4-VP as monomer. Also, the molar ratio of template and monomer can affect the affinity and imprinting effect of dt-MIPs toward the templates. ${ }^{43}$ Molar ratios of template and monomer of $1: 2$ and 1:4 were investigated, while the molar ratio of template to cross-linker (EGDMA) was set at $1: 20$. The dt-MIPs prepared using a molar ratio of template/ monomer/cross-linker of $1: 4: 20$ showed excellent adsorption affinity for NOR and ENR. Therefore, under the optimized preparation conditions, new dt-MIPs were prepared by simple and facile precipitation polymerization.

\section{Characterization of dt-MIPs}

The as-prepared dt-MIPs were characterized by SEM, BET, FT-IR and TGA as follows. Fig. 2A shows the SEM images of dtMIPs (a) and NIPs (b), demonstrating that uniform and spherical particles, relatively rough surfaces and smaller average diameters were observed for the MIPs. As seen from Fig. 2B, the dt-MIPs and NIPs exhibited good size distribution, and the dominant distribution peak was at about $190 \mathrm{~nm}$ for dt-MIPs (a) and $350 \mathrm{~nm}$ for NIPs (b), possibly resulting from the influence of the template on particle growth during the precipitation polymerization. ${ }^{44}$ This phenomenon might be attributed to different forms of MAA in the dt-MIP and NIP reaction systems. During the preparation of NIPs, MAA could form hydrogen-bonded dimers in the absence of template, and both free MAA and MAA dimers were present in the pre-polymerization solution. In the dt-MIP reaction system, additional molecular interactions between MAA and the template were formed, which might affect the growth of the cross-linked polymer nuclei and result in smaller polymer particles. ${ }^{45}$

Surface area and pore volume are important parameters that affect the adsorption capacity of imprinted polymers. By BET analysis, the specific surface area of the dt-MIPs was $48.73 \mathrm{~m}^{2} \mathrm{~g}^{-1}$, much larger than that of the corresponding NIPs $\left(14.40 \mathrm{~m}^{2} \mathrm{~g}^{-1}\right)$, probably leading to a higher adsorption capacity for the dt-MIPs. Likewise, the dt-MIPs possessed much larger pore volume $\left(0.43 \mathrm{~mL} \mathrm{~g}^{-1}\right)$ than the NIPs $(0.18 \mathrm{~mL}$ $\mathrm{g}^{-1}$ ), which might be attributed to the formation of binding cavities on the surface of the dt-MIPs. Moreover, the dt-MIPs and NIPs exhibited average pore sizes of 24.84 and $21.77 \mathrm{~nm}$, respectively. According to the commonly used classification of the International Union of Pure and Applied Chemistry (IUPAC), the pores should be classified as mesopores (between $2 \mathrm{~nm}$ and $50 \mathrm{~nm}$ ). The mesopores can also affect the polymers' adsorption properties to some degree. 
A

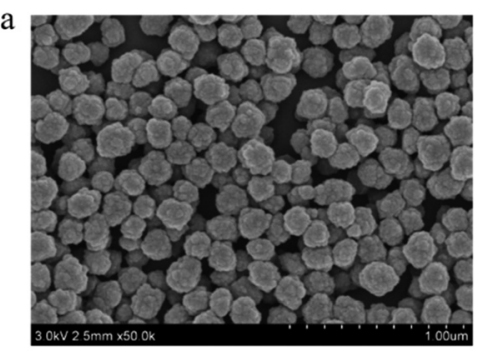

B a

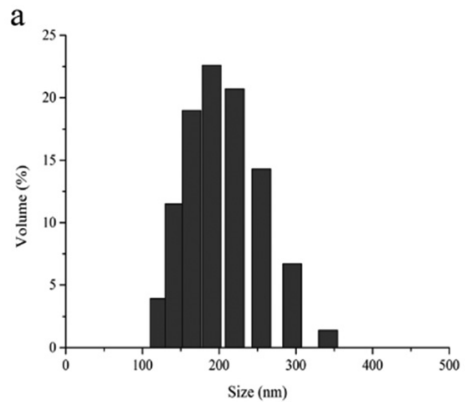

b

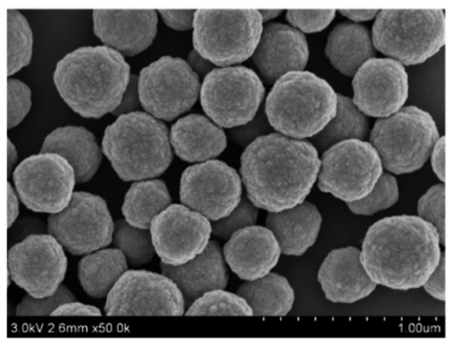

b

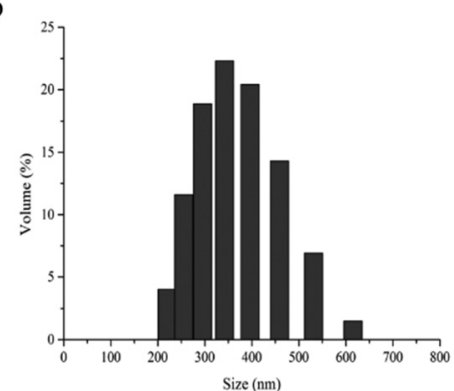

Fig. 2 (A) SEM images of dt-MIPs (a) and NIPs (b) at the same scale $(1 \mu \mathrm{m})$. (B) Particle size distribution profiles of dt-MIPs (a) and NIPs (b).

FT-IR spectra of the dt-MIPs before and after template removal are shown in Fig. 3A. It can be seen from curves a and $\mathrm{b}$ that strong absorption bands at around $3428 \mathrm{~cm}^{-1}$ and $1731 \mathrm{~cm}^{-1}$ were observed, which could be ascribed to the $\mathrm{O}-\mathrm{H}$ and $\mathrm{C}=\mathrm{O}$ stretching vibrations of the carboxylic acid group of MAA. The significant absorption bands at around $1257 \mathrm{~cm}^{-1}$ and $1157 \mathrm{~cm}^{-1}$ could be assigned to the symmetric and asymmetric $\mathrm{C}-\mathrm{O}$ stretching vibrations of EGDMA. ${ }^{29}$ The peaks at $2985 \mathrm{~cm}^{-1}$ and $2954 \mathrm{~cm}^{-1}$ were attributed to the C-H stretching vibrations of $-\mathrm{CH}_{3}$ and $-\mathrm{CH}_{2}$ groups. Compared with the IR spectra of NOR (curve c) and ENR (curve d), the C-F stretching vibration at $960 \mathrm{~cm}^{-1}$ disappeared and the $\mathrm{C}=\mathrm{C}$ vibration of the benzene ring at around $1465 \mathrm{~cm}^{-1}$ became weak after template removal (curve b), which could indicate that the dtMIPs were successfully prepared.
TGA was used to evaluate the thermal stability of the dtMIPs. As shown in Fig. 3B, the dt-MIPs exhibited a slight weight loss with an increase in temperature from $40{ }^{\circ} \mathrm{C}$ to $220{ }^{\circ} \mathrm{C}$, which might be attributed to water evaporation. ${ }^{46}$ At temperatures ranging from $220{ }^{\circ} \mathrm{C}$ to $450{ }^{\circ} \mathrm{C}$, a rapid weight loss occurred, indicating decomposition of the material. Then, the weight of the dt-MIPs tended to stabilize at higher temperature, with the full release of a volatile compound. Therefore, the synthesized dt-MIPs showed good thermal stability at temperatures lower than $220{ }^{\circ} \mathrm{C}$. This phenomenon can also be clearly observed from the corresponding DTG curve (Fig. 3B).

\section{Adsorption analysis}

The adsorption capacities of the dt-MIPs and NIPs for NOR and ENR were evaluated by static adsorption isotherms, as
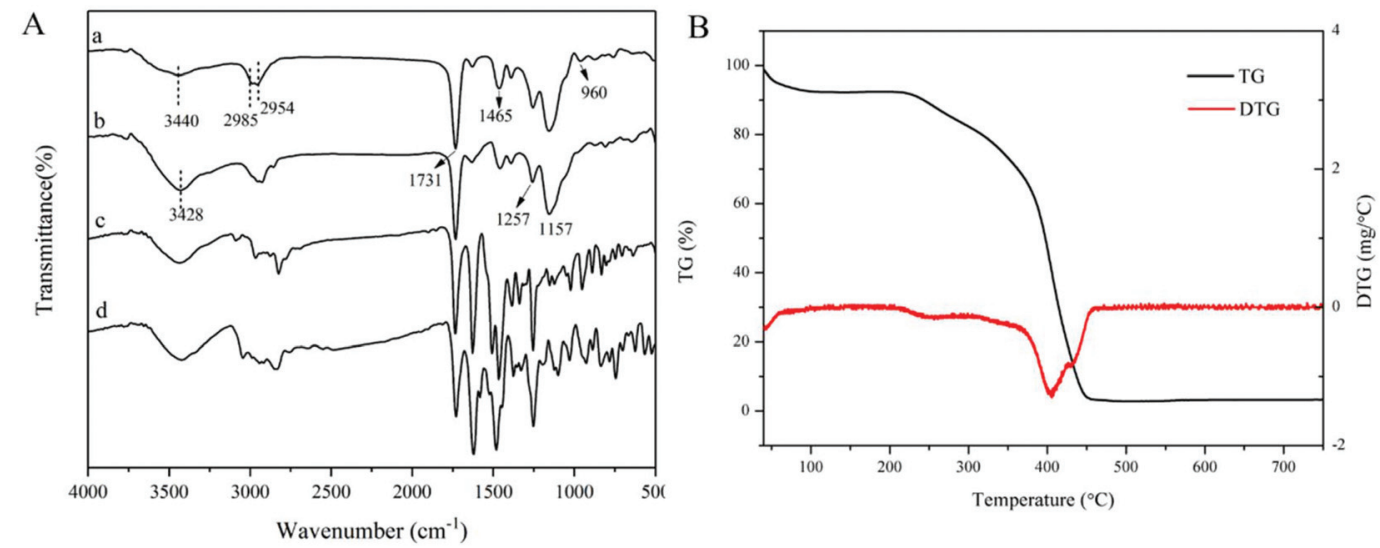

Fig. 3 (A) FT-IR spectra of dt-MIPs before (a) and after (b) template removal, and spectra of NOR (c) and ENR (d). (B) TG/DTG curves of dt-MIPs. 
shown in Fig. S1.† As seen from the figure, for the dt-MIPs, the adsorption capacities for NOR and ENR increased continuously with an increase in initial concentration and then became stable above the equilibrium concentration of $80 \mathrm{mg} \mathrm{L}{ }^{-1}$, while the NIPs exhibited a similar trend but lower binding capacities. The maximum adsorption capacities of the dt-MIPs for NOR and ENR were 32.0 and $21.8 \mathrm{mg} \mathrm{g}^{-1}$, respectively. Whereas, the maximum adsorption capacities of the NIPs were lower, $14.0 \mathrm{mg} \mathrm{g}^{-1}$ and $13.1 \mathrm{mg} \mathrm{g}^{-1}$ for NOR and ENR, respectively. Based on the principle of adsorption-desorption equilibrium between polymers and templates, the templates can only occupy a small portion of the recognition sites of the dt-MIPs at low initial concentration, resulting in a low adsorption capacity. With an increase in concentration, the recognition sites of the dt-MIPs were gradually occupied by templates, so that the adsorption capacity increased sharply and reached saturation. Because of the absence of specific recognition sites in the NIPs, a comparatively lower adsorption capacity was observed due to the dominating physical adsorption. On the other hand, the maximum adsorption capacity of the dt-MIPs was higher for NOR than for ENR, which might be ascribed to the different interaction strength between each template and the functional monomer. As a result, the synthesized dt-MIPs could selectively recognize and adsorb the two template compounds simultaneously. And the dt-MIPs with high adsorption capacity would achieve high extraction efficiency for the trace analysis of NOR and ENR.

To estimate the selectivity of the dt-MIPs for NOR and ENR, four other structurally related FQs (PEF, DAN, ENO and CIP) were chosen and investigated. The chemical structures of the FQs used in this study and the adsorption capacities of the dtMIPs and NIPs for them are shown in Fig. S2 and S3, $\dagger$ respectively. As seen from Fig. $\mathrm{S} 3, \dagger$ similar low adsorption capacities of dt-MIPs and NIPs for PEF, DAN, ENO and CIP were attained. The $p$ values from the Student's $t$-test were all more than 0.05 , which illustrated that there were no statistically significant differences. The above mentioned data confirmed that nonspecific adsorption possibly occurred between the structural analogues and the dt-MIPs/NIPs. In contrast, the adsorption capacities of the dt-MIPs were much higher for the two templates, and the $p$ values were lower than 0.01 , which was considered as a highly significant difference, possibly resulting from the fact that specific recognition sites for NOR and ENR in terms of shape, size and functionality were generated on the dt-MIPs. Also, the imprinting factors $(\alpha)$, distribution coefficients $\left(K_{\mathrm{d}}\right)$, selectivity coefficients $(K)$ and relative selectivity coefficients $\left(K^{\prime}\right)$ of the templates and analogues on dt-MIPs and NIPs were calculated for selectivity evaluation, as summarized in Table S1.† As shown in the table, good imprinting effects were indicated by the higher imprinting factors for NOR and ENR than for the analogues. The $K_{\mathrm{d}}$ values of the dtMIPs for NOR and ENR were greater than those of the NIPs, which demonstrated that the distribution of imprinted cavities/recognition sites in the dt-MIPs was ordered according to a predetermined orientation. The $K$ values of the dt-MIPs for NOR and ENR were significantly larger than those of the NIPs, implying that the dt-MIPs had higher recognition selectivity for the two templates. The $K^{\prime}$ values indicated an enhancement in adsorption affinity and selectivity for the template molecules on the dt-MIPs with respect to the NIPs. The difference in binding ability between the two templates and the other analogues on the dt-MIPs revealed that the geometric affinity played an essential role in the selectivity of the dt-MIPs. The above results confirmed that the dt-MIPs had higher recognition and binding affinity for NOR and ENR than the NIPs, and that the dt-MIPs could highly selectively extract these two templates simultaneously. Moreover, it was found that the adsorption capacities of the NIPs for the two templates, NOR and ENR, were much larger than for the four structural analogues (Fig. S3†). For the selective adsorption experiments, the adsorption capacities of the NIPs for the templates and the structural analogues are mainly influenced by the adsorption performance of the NIPs, the adsorption conditions and the physicochemical properties of the analytes. ${ }^{4-50}$ In our study, considering that the adsorption experiments were carried out under the same conditions, the adsorption capacities of the NIPs for the FQs should be related to the pore structure and specific surface area of the NIPs, and to the steric hindrance and water solubility of the FQs.

\section{Optimization of dt-MIPs-DSPE conditions}

The proposed dt-MIPs with spherical morphology and high adsorption capacity were used as adsorbents for DSPE, coupled with HPLC-DAD, for simultaneous extraction and determination of NOR and ENR in aqueous solutions. Several main parameters affecting the extraction efficiency of dt-MIPsDSPE were investigated in detail and optimized, including dtMIP dosage, sample $\mathrm{pH}$, type and volume of desorption solvent, and extraction and desorption time. All experiments were performed in triplicate using a standard aqueous solution with individual NOR and ENR concentrations of $50 \mu \mathrm{g}$ $\mathrm{L}^{-1}$.

Effect of dt-MIP dosage. One of the advantages of DSPE is that high extraction efficiency can be achieved by using smaller amounts of adsorbent than in traditional SPE. To investigate the effect of dt-MIP dosage, 3 to $15 \mathrm{mg}$ dt-MIPs were dispersed into a $10 \mathrm{~mL}$ neutral aqueous solution containing $50 \mu \mathrm{g} \mathrm{L}^{-1}$ NOR and ENR by ultrasonication. After extraction for $3 \mathrm{~h}$ at room temperature, $150 \mu \mathrm{L}$ methanol was used to desorb the analytes under ultrasound for $5 \mathrm{~min}$. The results are shown in Fig. 4A; the extraction efficiency increased on increasing the dosage from 3 to $10 \mathrm{mg}$, and then there was almost no change at $15 \mathrm{mg}$, indicating that $10 \mathrm{mg}$ dt-MIPs were sufficient for the extraction of ENR and NOR in aqueous solution. Therefore, $10 \mathrm{mg} \mathrm{dt}$-MIPs were used for further studies.

Effect of sample pH. Since FQs are amphoteric compounds containing amino and carboxylic groups, sample $\mathrm{pH}$ is a crucial factor that influences the form present in aqueous solution and the adsorption capabilities of dt-MIPs. Through an extensive literature review, it was found that different $\mathrm{p} K_{\mathrm{a}}$ values of FQs have been reported by different researchers 

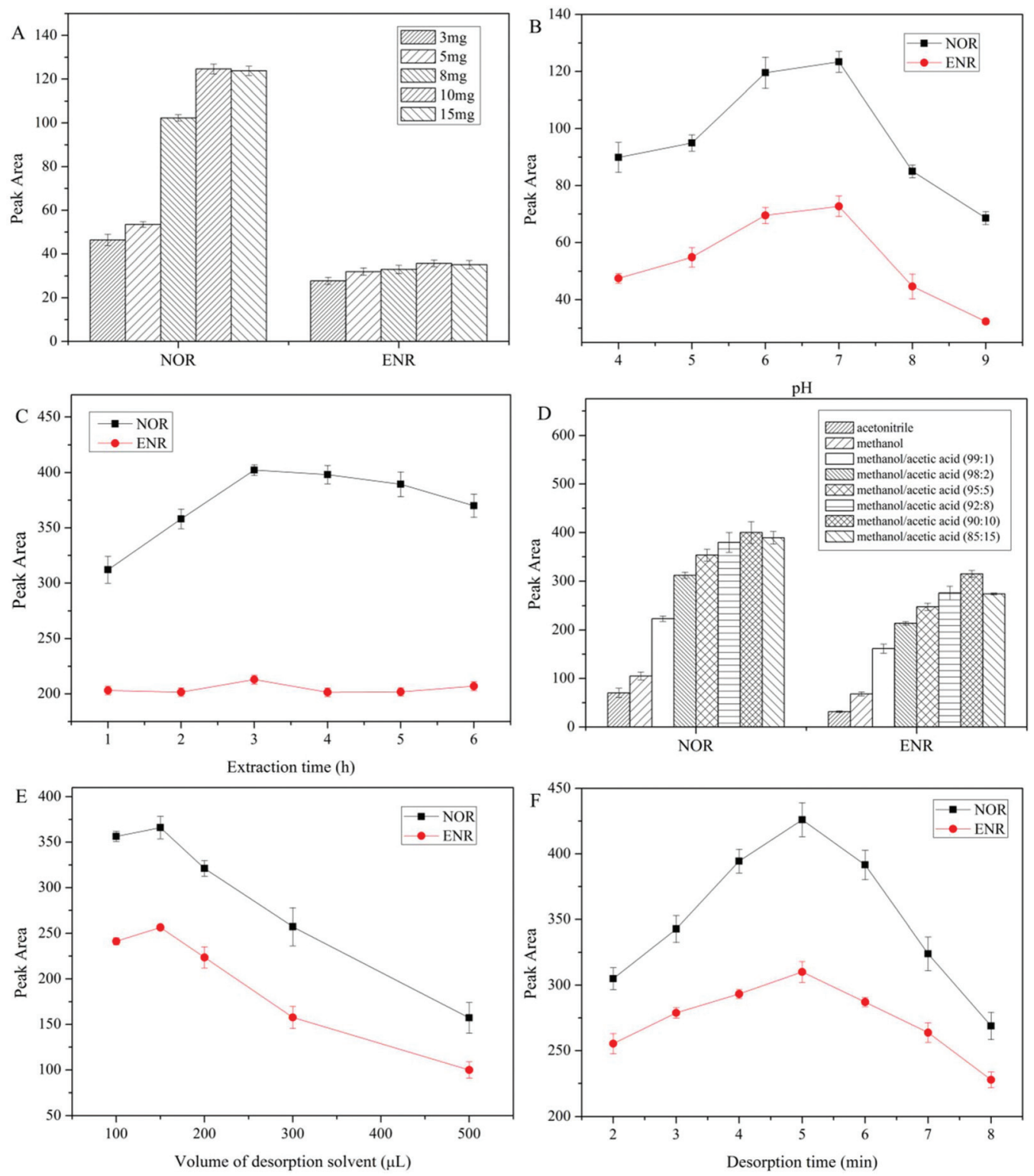

Fig. 4 Effect of dt-MIP dosage (A), sample pH (B), extraction time (C), type of desorption solvent (D), volume of desorption solvent (E) and desorption time (F) on the extraction performance of dt-MIPs-DSPE. Extraction conditions: sample volume, $10.0 \mathrm{~mL}$; (A) sample pH, 7.0; extraction time, $3 \mathrm{~h}$; desorption solvent and volume, $150 \mu \mathrm{L}$ methanol; desorption time, $5 \mathrm{~min}$; (B) dt-MIP dosage, $10 \mathrm{mg}$; extraction time, $3 \mathrm{~h}$; desorption solvent and volume, $150 \mu \mathrm{L}$ methanol; desorption time, $5 \mathrm{~min}$; (C) dt-MIP dosage, $10 \mathrm{mg}$; sample $\mathrm{pH}$, 7.0; desorption solvent and volume, $150 \mu \mathrm{L}$ methanol containing 10\% acetic acid (v/v); desorption time, $5 \mathrm{~min}$; (D) dt-MIP dosage, $10 \mathrm{mg}$; sample $\mathrm{pH}$, 7.0; extraction time, $3 \mathrm{~h}$; desorption volume, 150 LL; desorption time, $5 \mathrm{~min}$; (E) dt-MIP dosage, $10 \mathrm{mg}$; sample $\mathrm{pH}$, 7.0; extraction time, $3 \mathrm{~h}$; desorption solvent, methanol containing $10 \%$ acetic acid $(\mathrm{v} / \mathrm{v})$; desorption time, $5 \mathrm{~min}$; (F) dt-MIP dosage, $10 \mathrm{mg}$; sample $\mathrm{pH}, 7.0$; extraction time, $3 \mathrm{~h}$; desorption solvent and volume, $150 \mathrm{\mu L}$ methanol containing $10 \%$ acetic acid $(\mathrm{v} / \mathrm{v})$.

using different determination methods. ${ }^{51}$ For NOR, the $\mathrm{p} K_{\mathrm{a} 1}$ (carboxylic acid) and $\mathrm{p} K_{\mathrm{a} 2}$ (nitrogen atom on the piperazine ring) values have been reported to be 5.85-6.34 and 8.10-10.17, respectively. ${ }^{51-54}$ For ENR, the $\mathrm{p} K_{\mathrm{a} 1}$ and $\mathrm{p} K_{\mathrm{a} 2}$ values corresponding to the carboxylic acid and the nitrogen atom on the piperazine ring are reported to be 5.88-6.81 and 7.70-8.04, respectively. ${ }^{51-53,55}$ In view of the $\mathrm{p} K_{\mathrm{a}}$ values, $\mathrm{pH}$ values of FQ solutions ranging from 4.0-9.0 were evaluated by adjusting with $1 \mathrm{~mol} \mathrm{~L}^{-1} \mathrm{HCl}$ or $1 \mathrm{~mol} \mathrm{~L}^{-1} \mathrm{NaOH}$. As shown in
Fig. 4B, the extraction efficiency for NOR and ENR increased gradually with an increase in sample $\mathrm{pH}$ from 4.0 to 7.0 and then decreased at alkaline $\mathrm{pH}$. The highest values were obtained at $\mathrm{pH}$ 7.0, which might be attributed to the strong binding affinity of dt-MIPs for FQs present as the zwitterionic form. ${ }^{51}$ Meanwhile, the presence of NOR and ENR as cationic forms at low $\mathrm{pH}$ (acidic) could be ascribed to the protonation of amino groups, and decreased the molecular recognition ability of the imprinting sites for NOR and ENR. Similarly, at 
high pH (alkaline), MAA and the FQs were present in their anionic forms, resulting in a decrease in the adsorbed amounts because of repulsive electrostatic interactions. ${ }^{42}$ Therefore, the sample $\mathrm{pH}$ was set at 7.0, which was convenient, feasible and highly desirable for real water treatment.

Effect of extraction time. Extraction time is an important factor to ensure the sufficient adsorption of the target analytes by MIPs. For the present dt-MIPs-DSPE, extraction times ranging from 1 to $6 \mathrm{~h}$ were examined. As shown in Fig. 4C, the extraction efficiency increased gradually from 1 to $3 \mathrm{~h}$, decreased from 3 to $4 \mathrm{~h}$, followed by almost identical values within 4-5 h, and then increased from 5 to $6 \mathrm{~h}$ without an obvious increase compared with that at $3 \mathrm{~h}$. Consequently, $3 \mathrm{~h}$ was chosen as the extraction time, since adsorption equilibrium between the FQs and the dt-MIPs could be reached along with high efficiency.

Effect of desorption conditions. In order to fully desorb the two FQs retained in the dt-MIPs, type and volume of desorption solvent and desorption time were investigated in detail. Firstly, eight kinds of desorption solvents, including acetonitrile, methanol and methanol containing 1, 2, 5, 8, 10 and $15 \%$ acetic acid $(\mathrm{v} / \mathrm{v})$, were used to optimize the desorption conditions. As shown in Fig. 4D, the results indicated that methanol showed better extraction efficiency than acetonitrile and the addition of acetic acid significantly increased the desorption efficiency. A possible reason is that acetic acid competing with the FQs for the functional groups in the binding sites of the dt-MIPs could break the hydrogen bonding interactions between the FQs and the dt-MIPs. However, more acetic acid did not improve desorption, and resulted in a decrease in extraction efficiency (Fig. 4D). Therefore, methanol containing $10 \%$ acetic acid (v/v) was selected as the desorption solvent for subsequent experiments.

Volumes of desorption solvent ranging from 100 to $500 \mu \mathrm{L}$ were also investigated, and the maximum extraction efficiency was attained using $150 \mu \mathrm{L}$, as obviously seen from Fig. 4E. The FQs bound in the dt-MIPs could not be desorbed sufficiently using a lower volume of elution solvent, while larger volumes reduced the extraction efficiency. Thus, $150 \mu \mathrm{L}$ methanol/ acetic acid ( $90: 10, \mathrm{v} / \mathrm{v})$ was employed for desorption.

The effect of desorption time on the extraction efficiency of FQs was studied for a range of 2-8 min. As observed in Fig. 4F, with an extension of desorption time, the extraction efficiency increased gradually and reached a maximum at $5 \mathrm{~min}$, and then decreased. This indicated that 5 min was enough time to desorb the retained FQs from the dt-MIPs, whereas longer ultrasonic desorption times would make a small volume of the desorption solvent splash on the centrifuge tube wall, resulting in a decline in extraction efficiency. Accordingly, the optimal conditions for desorption were set as using $150 \mu \mathrm{L}$ methanol/ acetic acid (90:10, v/v) and a desorption time of $5 \mathrm{~min}$.

\section{Method validation and practical application of the dt-MIPs- DSPE}

The selective dt-MIPs-DSPE coupled with HPLC was validated by investigating linearity, limit of detection (LOD), limit of quantification (LOQ), intraday/interday precision, and extraction recovery (ER), as well as the enrichment factor (EF). The equations for EF and ER are shown in Experimental S2 $\uparrow$ and all the results are presented in Table 1 . As seen, good linearity was obtained in the range of $1-200 \mu \mathrm{g} \mathrm{L} \mathrm{L}^{-1}$, with correlation coefficients ( $r$ ) of 0.9977 and 0.9989 for NOR and ENR, respectively. The LOD and LOQ were 0.22 and $0.67 \mu \mathrm{g} \mathrm{L}^{-1}$ for NOR, respectively, and 0.36 and $0.98 \mu \mathrm{g} \mathrm{L}^{-1}$ for ENR, obtained based on the signal-to-noise ratio $(\mathrm{S} / \mathrm{N}=3$ and 10 , respectively). The intraday and interday precision are expressed as the relative standard deviations (RSDs), which were calculated via five replicate analyses at $50 \mu \mathrm{g} \mathrm{L}^{-1}$ on the same day and five different days, respectively. Values of 3.0 and $7.3 \%$ respectively were obtained for NOR, and values of 3.3 and $4.3 \%$ respectively were obtained for ENR. The RSD values suggest that the method is highly accurate and reliable. The high EF and extraction recovery values (Table 1 ) fully demonstrate the highly selective pretreatment ability of the dt-MIPs-DSPE, which enabled the highly sensitive and concurrent determination of the two FQs.

Furthermore, the validated DSPE method was applied to real water sample analysis including lake water, sea water and tap water. Endogenous FQs were not detected in the three water samples, owing to their quite low content in complicated matrices. Whereas, for water samples spiked with NOR and ENR at three concentration levels of 5,20 and $50 \mu \mathrm{g} \mathrm{L}^{-1}$, the two FQs were easily found with complete base separation in less than $7 \mathrm{~min}$, which could be attributed to the selective recognition ability and high adsorption capacity of the as-prepared dt-MIPs. Taking the samples spiked at $50 \mu \mathrm{g} \mathrm{L}^{-1}$ as examples, as shown in Fig. $\mathrm{S} 4, \dagger$ there are no obvious matrix interferences in the extraction and analysis of all three real water samples. Moreover, as listed in Table 2, satisfactory recoveries of the above three concentrations of FQs in the

Table 1 Analytical performance of the dt-MIPs-DSPE-HPLC method for the determination of the two FQs

\begin{tabular}{|c|c|c|c|c|c|c|c|c|c|}
\hline FQ & Calibration curve $^{a}$ & $\begin{array}{l}\text { Correlation } \\
\text { coefficient }(r)\end{array}$ & $\begin{array}{l}\text { Linear } \\
\text { range } \\
\left(\mu g L^{-1}\right)\end{array}$ & $\begin{array}{l}\text { LOD } \\
\left(\mu g \mathrm{~L}^{-1}\right)\end{array}$ & $\begin{array}{l}\text { LOQ } \\
\left(\mu g \mathrm{~L}^{-1}\right)\end{array}$ & $\begin{array}{l}\text { Intraday } \\
\text { precision }^{b} \\
(\mathrm{RSD}, \%)\end{array}$ & $\begin{array}{l}\text { Interday } \\
\text { precision }^{b} \\
(\mathrm{RSD}, \%)\end{array}$ & $\mathrm{EF}^{b}$ & $\mathrm{ER}^{b}(\%)$ \\
\hline NOR & $y=(2.48 \pm 0.07) x+(89.9 \pm 6.85)$ & 0.9977 & $1-200$ & 0.22 & 0.67 & 3.0 & 7.3 & 71 & 106.4 \\
\hline ENR & $y=(2.44 \pm 0.05) x+(44.3 \pm 4.74)$ & 0.9989 & $1-200$ & 0.36 & 0.98 & 3.3 & 4.3 & 61 & 91.2 \\
\hline
\end{tabular}

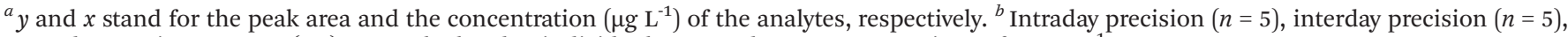
EF and extraction recovery (ER) were calculated at individual NOR and ENR concentrations of $50 \mu \mathrm{g} \mathrm{L}^{-1}$. 
Table 2 Recovery of the two FQs in spiked water samples by the developed dt-MIPs-DSPE-HPLC method $(n=3)$

\begin{tabular}{|c|c|c|c|c|c|c|c|c|c|c|}
\hline \multirow[b]{2}{*}{ FQ } & \multirow[b]{2}{*}{$\begin{array}{l}\text { Spiked } \\
\text { concentration } \\
\left(\mu \mathrm{g} \mathrm{L}^{-1}\right)\end{array}$} & \multicolumn{3}{|l|}{ Lake water } & \multicolumn{3}{|l|}{ Sea water } & \multicolumn{3}{|l|}{ Tap water } \\
\hline & & $\begin{array}{l}\text { Average recovered } \\
\text { concentration } \pm \\
\mathrm{SD}^{a}\left(\mu \mathrm{g} \mathrm{L}{ }^{-1}\right)\end{array}$ & $\begin{array}{l}\text { Recovery } \\
(\%)\end{array}$ & $\begin{array}{l}\text { RSD } \\
(\%)\end{array}$ & $\begin{array}{l}\text { Average recovered } \\
\text { concentration } \pm \\
\mathrm{SD}\left(\mu \mathrm{g} \mathrm{L}^{-1}\right)\end{array}$ & $\begin{array}{l}\text { Recovery } \\
(\%)\end{array}$ & $\begin{array}{l}\text { RSD } \\
(\%)\end{array}$ & $\begin{array}{l}\text { Average recovered } \\
\text { concentration } \pm \\
\mathrm{SD}\left(\mu \mathrm{g} \mathrm{L}^{-1}\right)\end{array}$ & $\begin{array}{l}\text { Recovery } \\
(\%)\end{array}$ & RSD (\%) \\
\hline \multirow[t]{3}{*}{ NOR } & 5.0 & $4.8 \pm 0.3$ & 97.0 & 5.6 & $5.0 \pm 0.1$ & 99.1 & 1.2 & $5.0 \pm 0.1$ & 99.3 & 2.3 \\
\hline & 20.0 & $16.2 \pm 0.1$ & 81.2 & 0.9 & $16.7 \pm 0.6$ & 83.7 & 3.5 & $19.4 \pm 0.7$ & 96.8 & 3.4 \\
\hline & 50.0 & $43.8 \pm 0.9$ & 87.6 & 2.0 & $46.7 \pm 2.6$ & 93.3 & 5.6 & $43.0 \pm 1.6$ & 86.1 & 3.6 \\
\hline \multirow[t]{3}{*}{ ENR } & 5.0 & $5.0 \pm 0.1$ & 100.8 & 1.8 & $4.8 \pm 0.2$ & 97.1 & 3.7 & $5.0 \pm 0.2$ & 101.0 & 3.4 \\
\hline & 20.0 & $17.1 \pm 1.2$ & 85.5 & 6.9 & $16.2 \pm 0.2$ & 80.9 & 1.1 & $19.9 \pm 1.1$ & 99.4 & 5.4 \\
\hline & 50.0 & $47.9 \pm 1.6$ & 95.9 & 3.4 & $46.8 \pm 3.1$ & 93.6 & 6.7 & $48.8 \pm 2.6$ & 97.5 & 5.4 \\
\hline
\end{tabular}

three kinds of water samples were attained, i.e., 80.9-101.0\% with RSDs of $0.9-6.9 \%$. For instance, in the sea water samples, the recoveries ranged from 83.7 to $99.1 \%$ with RSDs of $1.2-5.6 \%$ for NOR, and from 80.9 to $97.1 \%$ with RSDs of $1.1-6.7 \%$ for ENR. Consequently, the dt-MIP based DSPE method was shown to be practically feasible for effective enrichment, separation and determination of NOR and ENR simultaneously in complicated water samples. And therefore, a convenient and cost-effective method was successfully proposed and developed for the simultaneous selective analysis and abatement of more than one target analyte at trace levels in complicated matrices.

\section{Method performance comparison}

The analytical performance of this developed method was compared with that of reported MIP based SPE-HPLC methods for the extraction of FQs, as listed in Table $22 . \dagger^{28,29,47,56,57}$ As seen from the table, our dt-MIPs were prepared by a simpler polymerization method than other reported MIPs. ${ }^{29,47,56,57}$ Although a one step preparation process was used to synthesize the polymers for both bulk polymerization and precipitation polymerization, the subsequent treatment procedure was complicated for bulk polymerization. The obtained bulk polymers needed to be crushed, ground and sieved before use in SPE, ${ }^{28}$ even though precipitation polymerization used more porogen. For surface imprinting, ${ }^{56,57}$ two or more preparation steps were required to synthesize the MIPs, which needed a longer preparation time and expended more organic solvents. For instance, magnetic MIPs exhibited high adsorption capacity and thereby achieved fast and selective magnetic extraction of FQs from biological fluids, however the sensitivity was lower than that of our MIPs, and relatively complex surface imprinting was required. ${ }^{47}$ Using the same precipitation polymerization for the MIPs, ${ }^{28}$ a lower amount of adsorbent was needed for our DSPE procedure, which achieved a high extraction efficiency comparable to that of traditional SPE. On the whole, our developed dt-MIPs-DSPE-HPLC method demonstrated high selectivity and sensitivity, simple and convenient operation, rapid concurrent determination of the FQs and good practical applicability.

\section{Conclusions}

To conclude, novel dt-MIPs were prepared by a precipitation polymerization and multi-template imprinting strategy using NOR and ENR as templates, and employed as DSPE adsorbents coupled with HPLC-DAD for simultaneous selective extraction and determination of two FQs in environmental water samples. The developed simple, rapid and cost-effective dtMIPs-DSPE-HPLC method proved to be highly sensitive and practically applicable for the simultaneous selective determination of trace FQ antibiotics in complex aqueous samples.

On the other hand, based on some published studies regarding dt-MIPs in sample pretreatment techniques, the feasibility of the dual/multi-template imprinting strategy has also been fully confirmed. By summarizing these reports on dt-MIPs, it can be found that the innovation and research content mainly lie in the synthesis methods for the dt-MIPs (including the choice of template, functional monomer, crosslinker, initiators and preparation methods), the sample pretreatment techniques (mode choice and condition optimization), the target analytes, and the applications in real samples. However, dual/multi-template imprinting strategy related studies still have great challenges and promising opportunities. Especially, the synthesis of functionalized dtMIPs (e.g. magnetic dt-MIPs ${ }^{37,38}$ ) and the utilization of dt-MIPs for more than two target analytes ${ }^{39,40}$ are highly appreciated and should be strongly promoted. Furthermore, we can foresee a rapid advance in the dual/multi-template imprinting strategy and wider applications of the resulting dt/mt-MIPs in preparative technologies, high-throughput contaminant analysis and abatement.

\section{Conflicts of interest}

There are no conflicts to declare.

\section{Acknowledgements}

This work was financially supported by the National Natural Science Foundation of China (21876199, 21477160, 41601525, 
21804010, 41776110), the Natural Science Foundation of Shandong Province of China (ZR2014BL031, ZR2016BL25, ZR2016DB07), and the Department of Science and Technology of Shandong Province of China (GG201709290055).

\section{Notes and references}

1 N. Janecko, L. Pokludova, J. Blahova, Z. Svobodova and I. Literak, Environ. Toxicol. Chem., 2016, 35, 2647-2656.

2 Q. T. Dinh, E. Moreau-Guigon, P. Labadie, F. Alliot, M. J. Teil, M. Blanchard and M. Chevreuil, Chemosphere, 2017, 168, 483-490.

3 M. Rusch, A. Spielmeyer, J. Meissner, M. Kietzmann, H. Zorn and G. Hamscher, J. Agric. Food Chem., 2017, 65, 3118-3126.

4 X. Van Doorslaer, J. Dewulf, H. Van Langenhove and K. Demeestere, Sci. Total Environ., 2014, 500-501, 250-269.

5 X. Liu, J. C. Steele and X. Z. Meng, Environ. Pollut., 2017, 223, 161-169.

6 M. M. Zheng, R. Gong, X. Zhao and Y. Q. Feng, J. Chromatogr. A, 2010, 1217, 2075-2081.

7 M. Denadai and Q. B. Cass, J. Chromatogr. A, 2015, 1418, 177-184.

8 S. Qu, X. Wang, C. Tong and J. Wu, J. Chromatogr. A, 2010, 1217, 8205-8211.

9 X. Liu, X. Wang, F. Tan, H. Zhao, X. Quan, J. Chen and L. Li, Anal. Chim. Acta, 2012, 727, 26-33.

10 V. H. Springer and A. G. Lista, Electrophoresis, 2015, 36, 1572-1579.

11 A. Speltini, F. Maraschi, R. Govoni, C. Milanese, A. Profumo, L. Malavasi and M. Sturini, J. Chromatogr. A, 2017, 1489, 9-17.

12 X. He, G. N. Wang, K. Yang, H. Z. Liu, X. J. Wu and J. P. Wang, Food Chem., 2017, 221, 1226-1231.

13 Y. J. Tang, J. Q. Xu, C. Le, J. L. Qiu, Y. Liu and G. F. Ouyang, Talanta, 2017, 175, 550-556.

14 H. Yan, F. Qiao and K. H. Row, Anal. Chem., 2007, 79, 8242-8248.

15 W. Y. Fan, M. He, X. R. Wu, B. B. Chen and B. Hu, J. Chromatogr. A, 2015, 1418, 36-44.

16 M. Mei and X. J. Huang, J. Sep. Sci., 2016, 39, 1908-1918.

17 M. R. Payan, M. A. B. Lopez, R. Fernandez-Torres, J. A. O. Gonzalez and M. C. Mochon, J. Pharm. Biomed. Anal., 2011, 55, 332-341.

18 I. Timofeeva, S. Timofeev, L. Moskvin and A. Bulatov, Anal. Chim. Acta, 2017, 949, 35-42.

19 Y. Zhao, L. Xu, M. Liu, Z. Duan and H. Wang, Food Chem., 2018, 239, 40-47.

20 Z. Duan, M. Yin, C. Zhang, G. Song, S. Zhao, F. Yang, L. Feng, C. Fan, S. Zhu and H. Wang, Analyst, 2018, 143, 392-395.

21 X. Zhu, Y. Cui, X. Chang and H. Wang, Talanta, 2016, 146, 358-363.

22 W. H. Lu, W. N. Ming, X. S. Zhang and L. X. Chen, Electrophoresis, 2016, 37, 2487-2495.
23 X. He, G. N. Wang, K. Yang, H. Z. Liu, X. J. Wu and J. P. Wang, Food Chem., 2017, 221, 1226-1231.

24 M. Amoli-Diva, K. Pourghazi and S. Hajjaran, Mater. Sci. Eng., C, 2016, 60, 30-36.

25 L. X. Chen, X. Y. Wang, W. H. Lu, X. Q. Wu and J. H. Li, Chem. Soc. Rev., 2016, 45, 2137-2211.

26 S. Farooq, J. Nie, Y. Cheng, Z. Yan, J. Li, S. A. S. Bacha, A. Mushtaq and H. Zhang, Analyst, 2018, 143, 3971-3989.

27 S. Xu, H. Lu and L. Chen, J. Chromatogr. A, 2014, 1350, 23-29.

28 E. Turiel, A. Martin-Esteban and J. L. Tadeo, J. Chromatogr. A, 2007, 1172, 97-104.

29 X. Wu and L. T. Wu, J. Sep. Sci., 2015, 38, 3615-3621.

30 J. L. Urraca, M. Castellari, C. A. Barrios and M. C. MorenoBondi, J. Chromatogr. A, 2014, 1343, 1-9.

31 A. C. Meng, J. LeJeune and D. A. Spivak, J. Mol. Recognit., 2009, 22, 121-128.

32 S. A. Zaidi, Electrophoresis, 2013, 34, 1375-1382.

33 R. X. Gao, Y. Hao, S. Q. Zhao, L. L. Zhang, X. H. Cui, D. C. Liu, Y. H. Tang and Y. S. Zheng, RSC Adv., 2014, 4, 56798-56808.

34 W. H. Lu, X. Y. Wang, X. Q. Wu, D. Y. Liu, J. H. Li, L. X. Chen and X. S. Zhang, J. Chromatogr. A, 2017, 1483, 30-39.

35 X. L. Song, J. H. Li, S. F. Xu, R. J. Ying, J. P. Ma, C. Y. Liao, D. Y. Liu, J. B. Yu and L. X. Chen, Talanta, 2012, 99, 75-82.

36 J. Liu, H. Song, J. Liu, Y. Liu, L. Li, H. Tang and Y. Li, Talanta, 2015, 134, 761-767.

37 M. Liu, X. Li, J. Li, Z. Wu, F. Wang, L. Liu, X. Tan and F. Lei, J. Colloid Interface Sci., 2017, 504, 124-133.

38 M. T. Jafari, B. Rezaei and H. Bahrami, Anal. Sci., 2018, 34, 297-303.

39 K. Yang, G. N. Wang, H. Z. Liu, J. Liu and J. P. Wang, J. Chromatogr. B: Anal. Technol. Biomed. Life Sci., 2017, 1046, 65-72.

40 W. Q. Xia, P. L. Cui, G. N. Wang, J. Li and J. P. Wang, Anal. Methods, 2018, 10, 3001-3010.

41 X. L. Song, J. H. Li, J. T. Wang and L. X. Chen, Talanta, 2009, 80, 694-702.

42 L. X. Chen, S. F. Xu and J. H. Li, Chem. Soc. Rev., 2011, 40, 2922-2942.

43 J. Qiao, H. Yan, H. Wang and Y. Lv, J. Sep. Sci., 2011, 34, 2668-2673.

44 P. Xiao, Y. Dudal, P. F. X. Corvini, P. Spahr and P. Shahgaldian, React. Funct. Polym., 2012, 72, 287-293.

45 F. Tan, D. M. Sun, J. S. Gao, Q. Zhao, X. C. Wang, F. Teng, X. Quan and J. W. Chen, J. Hazard. Mater., 2013, 244-245, 750-757.

46 H. L. de Oliveira, S. d. S. Anacleto, A. T. Maria da Silva, A. C. Pereira, W. d. S. Borges, E. C. Figueiredo and K. B. Borges, J. Chromatogr. B: Anal. Technol. Biomed. Life Sci., 2016, 1033-1034, 27-39.

47 D. Xiao, P. Dramou, N. Xiong, H. He, H. Li, D. Yuan and H. Dai, J. Chromatogr. A, 2013, 1274, 44-53.

48 T. Zhao, X. Guan, W. Tang, Y. Ma and H. Zhang, Anal. Chim. Acta, 2015, 853, 668-675. 
49 Y. Tang, J. Lan, X. Gao, X. Liu, D. Zhang, L. Wei, Z. Gao and J. Li, Food Chem., 2016, 190, 952-959.

50 P. Guo, X. Yuan, J. Zhang, B. Wang, X. Sun, X. Chen and L. Zhao, Anal. Bioanal. Chem., 2018, 410, 373389.

51 S. Babić, A. J. M. Horvat, D. Mutavdžić Pavlović and M. Kaštelan-Macan, TrAC, Trends Anal. Chem., 2007, 26, 1043-1061.

52 M. S. Burkhead, H. Wang, M. Fallet and E. M. Gross, Anal. Chim. Acta, 2008, 613, 152-162.
53 D. A. Volmer, B. Mansoori and S. J. Locke, Anal. Chem., 1997, 69, 4143-4155.

54 The Merck Index: An Encyclopedia of Chemicals, Drugs and Biologicals, 2006.

55 H. B. He, X. X. Lv, Q. W. Yu and Y. Q. Feng, Talanta, 2010, 82, 1562-1570.

56 X. L. Sun, X. M. Tian, Y. Z. Zhang and Y. J. Tang, Food Anal. Methods, 2013, 6, 1361-1369.

57 Y. K. Lv, Y. Ma, X. B. Zhao, C. L. Jia and H. W. Sun, Talanta, 2012, 89, 270-275. 